\title{
УДК 622.24 \\ ПРОГНОЗИРОВАНИЕ РИСКОВ ЗАКЛИНИВАНИЯ ДЛЯ ИСКЛЮЧЕНИЯ ВОЗМОЖНОСТИ ПРИХВАТОВ ТЕХНИЧЕСКОЙ СИСТЕМЫ «ПЕРФОБУР॥ ПРИ БУРЕНИИ РАЗВЕТВЛЕННЫХ КАНАЛОВ В ТЕРРИГЕННЫХ КОЛЛЕКТОРАХ
}

\author{
Лягов Илья Александрович', \\ ilyagov@perfobur.com
}

Губайдуллин Азат Гумарович², azat771989@mail.ru

\author{
Лягов Александр Васильевич ${ }^{2.1}$, \\ lyagovs@mail.ru
}
Могучев Александр Иванович², Moguchev@rusoil.net

\author{
Попов Анатолий Николаевич², \\ mongp2017@mail.ru

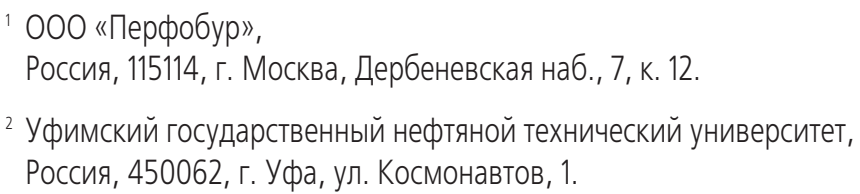

Актуальность. Одним из перспективных методов вторичного вскрытия продуктивных пород является бурение глубоких каналов малого диаметра технической системой радиального бурения «Перфобур». Рассмотрены вопросы прогнозирования и предупреждения прихватов бурильных компоновок технической системы вследствие возможного заклинивания в интервалах неустойчивых пород. Заклинивания в данных интервалах с большой степенью вероятности происходят вследствие упруговязкопластического деформирования поперечного сечения перфорационного канала.

Цель: оценка рисков прихватов технической системы «Перфобур» вследствие заклинивания при бурении и спускоподъемных операциях и разработка технологических мероприятий по их предупреждению.

объекты: глубокий канал малого диаметра и сверхмалого радиуса кривизны в терригенном пласте-коллекторе нефтяного месторождения в Поволжье, представляющего собой чередование аргиллита, алевролита и песчаника. Методы: геомеханическое моделирование, численное интегрирование, метод переменных модулей.

Результаты. Определены перемещения стенок глубокого перфорационного канала во времени для терригенного пласта-коллектора, представляющего собой чередование аргиллита, алевролита и песчаника. Получены зависимости упруговязкопластического перемещения стенок канала малого диаметра (58-60 мм) и радиуса кривизны (5-10 м) с изменением зенитного угла. Упруговязкопластическое перемещение верхней стенки перфорационного канала, как правило, значительно (в 1,5 и более раза) превышает упруговязкопластическое перемещение боковой стенки перфорационного канала. Следовательно, упруговязкопластическое перемещение верхней стенки является наиболее опасным и определяющим с точки зрения заклинивания особенно при спускоподъемных операциях. При бурении долотами типа PDC (опасное перемещение стенки равно 2 мм) определено, что наиболее опасным с точки зрения заклинивания является пропласток аргиллита и время безопасного ведения работ составляет один час. Для предупреждения возможного заклинивания компоновок технической системы «Перфобур» предложено применение специально разработанного секционного винтового забойного двигателя габарита 49 мм с кинематическим отношением рабочих органов - 9:10, что кратно увеличивает механическую скорость бурения по сравнению ранее применяемыми (также не серийными) винтовыми забойными двигателями с кинематикой рабочих органов - 5:6, использование струйных и волновых кольмататоров, расширителей, калибраторов-виброгасителей и осцилляторов, размещаемых в расчетных местах компоновки низа бурильной колонны для предотвращения и ликвидации возможных осложнений в радиальных каналах.

\section{Ключевые слова:}

Техническая система «Перфобур», компоновка низа бурильной колонны, прихват долота, упруговязкопластическое деформирование горной породы, призабойная зона пласта, бурение разветвленных радиальных каналов малого диаметра и радиуса кривизны.

\section{Введение}

В настоящее время интенсивно развиваются технологии вторичного вскрытия продуктивных пластов путем направленного бурения глубоких перфорационных каналов сверхмалого диаметра и радиуса кривизны с контролем траектории $[1,2]$. Одной из таких систем является техническая система «Перфобур» в модульном исполнении для радиального разветвленного бурения каналов по прогнозированной траектории из обсаженного ствола скважины, представленная на рис. 1 [3-6].

Среди возможных осложнений при работе технической системы «Перфобур» при бурении протяженных каналов для выхода компоновки низа бурильной колонны (КНБК) из зоны загрязнения продуктивной зоны пласта (ПЗП) следует выделить 


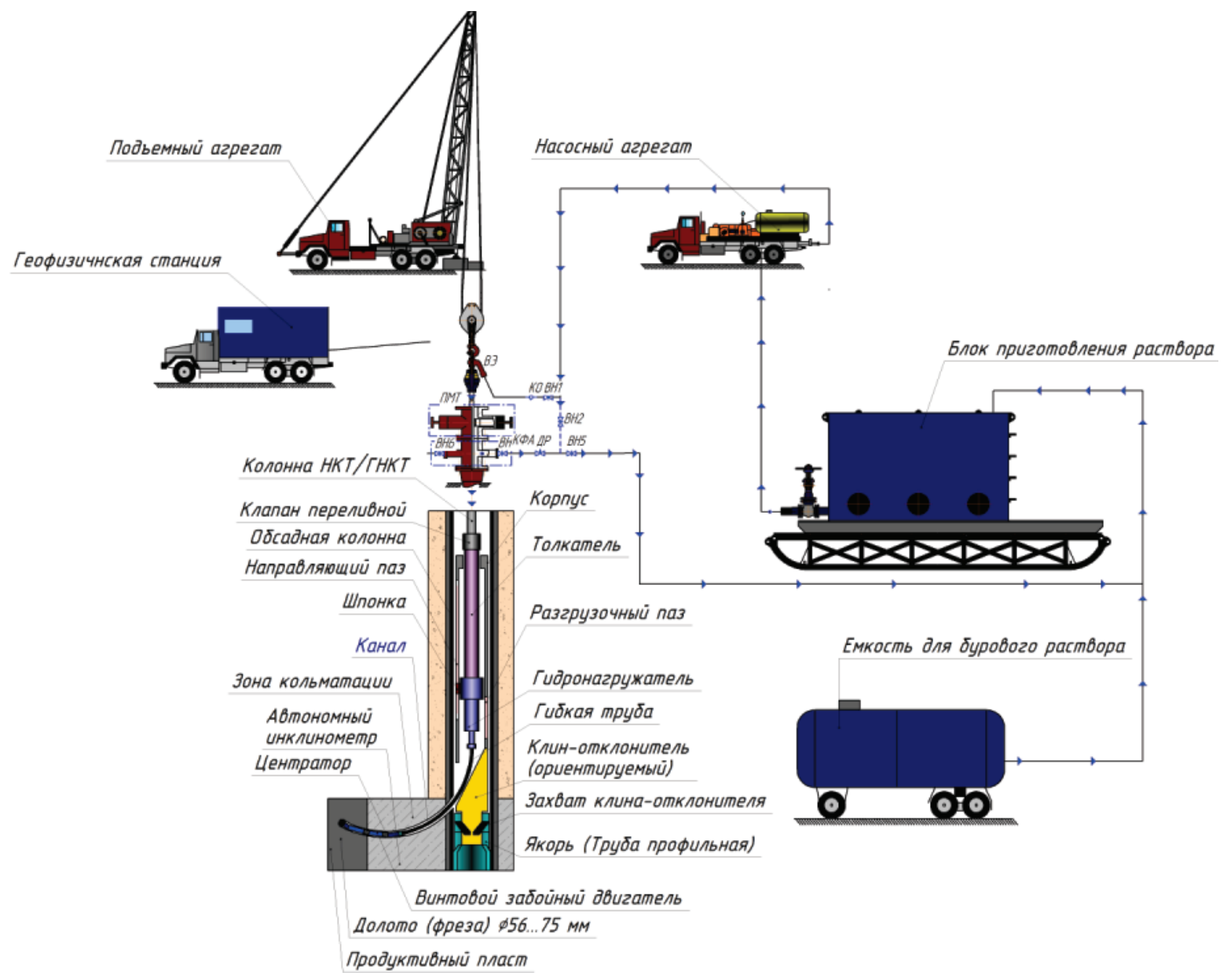

Pис. 1. Техническая система «Перфобур»

Fig. 1. «Perfobur» technical system

дифференциальные прихваты и прихваты вследствие заклинивания в местах деформирования поперечного сечения перфорационного канала. Причинами дифференциального прихвата являются: высокий перепад давления между стволом перфорационного канала и пластовым давлением, контакт бурильной колонны с проницаемой околоскважинной зоной, увеличение фильтрационной корки бурового раствора при остановке инструмента [7]. Признаками дифференциального прихвата является увеличение затяжек, посадок в начале движения колонны при нормальной циркуляции бурового раствора [8]. Первоначальными действиями для ликвидации осложнения являются изменение плотности бурового раствора и подбор режима работы осцилляторов, установленных в расчетных местах КНБК. Для предупреждения дифференциального прихвата компания «Перфобур» применяет следующие технологические меры (рис. 2):

1) поддержание оптимальной плотности бурового раствора;

2) установка в КНБК и поддержание устойчивой работы специальных осцилляторов при бурении в интервале возможного осложнения;
3) применение специальных КНБК с трубами различной частотной характеристики и забойным автоматом подачи долота (гидронагружателядемпфера);

4) контроль процесса бурения при вскрытии проницаемых зон [4, 9].

Однако вскрытие пород-коллекторов и породпокрышек с помощью радиальных перфорационных каналов сопровождается изменением их напряженно-деформированного состояния в околоскважинной зоне [10-21]. Вследствие этого происходит упруговязкопластическое деформирование поперечного сечения перфорационного канала, что в свою очередь может привести к заклиниванию долота при спускоподъемных операциях [22-27]. Данные проблемы устойчивости горных пород наиболее характерны для глинистых и хемогенных пород-покрышек, а также терригенных пород-коллекторов при наличии в них глинистых включений $[22,26]$. В крепких карбонатных породах реологические процессы происходят очень медленно, поэтому данные осложнения в них исключены [13].

В работах А.Н. Попова и др. рассмотрены вопросы прогнозирования деформирования попереч- 


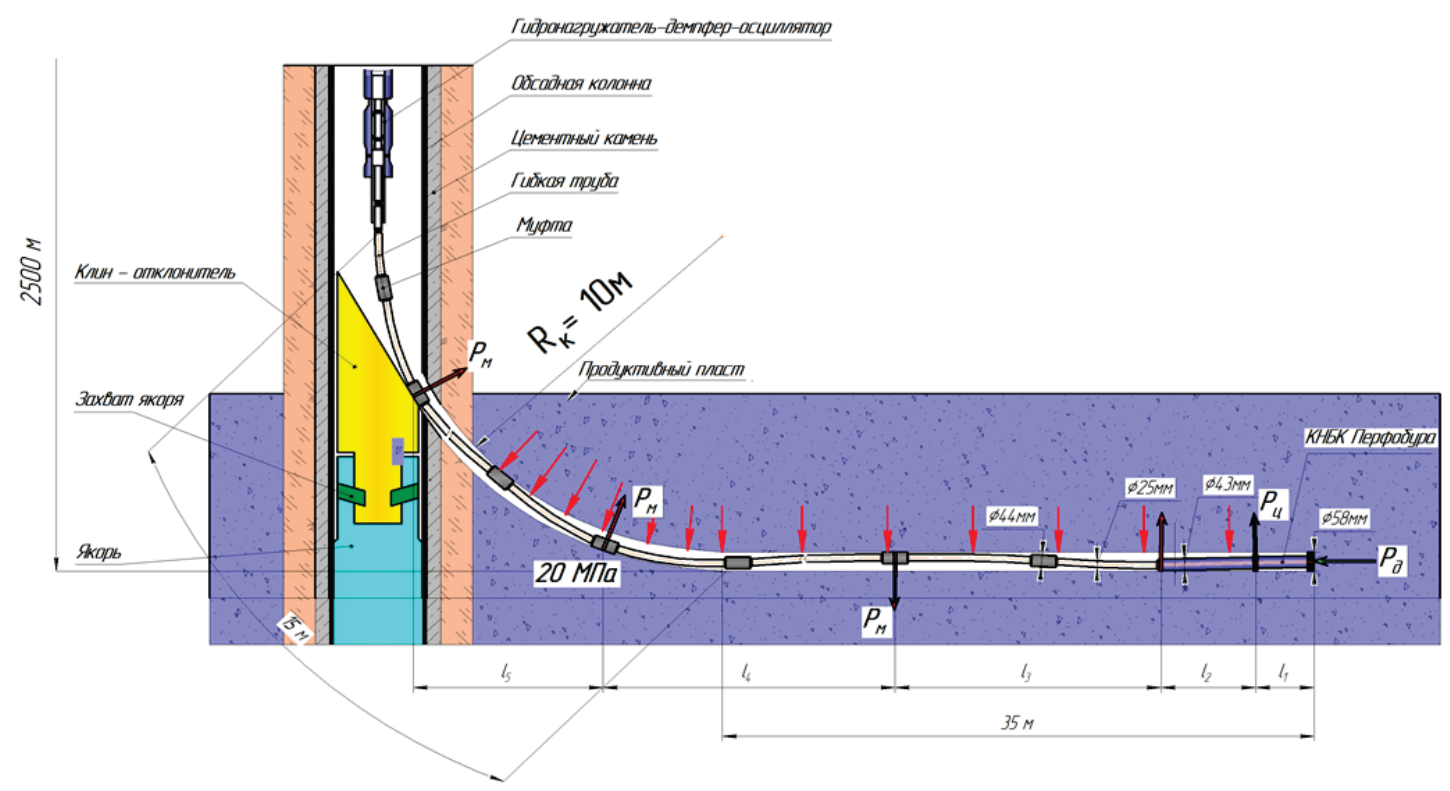

Рис. 2. Модель «стабилизирующей» КНБК «Перфобура» с осиилляторали при бурении канала общей длиной 50 м

Fig. 2. Stabilized stem assembly model of the «Perfobur» bottom hole assembly (BHA) completely with oscillators for drilling $50 \mathrm{~m}$ length channel

ного сечения открытого ствола наклонно направленных и горизонтальных скважин и снижения прихватоопасности долот вследствие заклинивания при подъеме из скважины [26, 27].

Глубокие перфорационные каналы, пробуренные технической системой «Перфобур», представляют собой, по сути, интервал траектории скважины малого диаметра. Это позволяет применить математическую модель упруговязкопластического перемещения стенок наклонно направленных и горизонтальных скважин с учетом сжимаемости горной породы, представленную в работе [26], для прогнозирования напряженно-деформированного состояния в окрестности перфорационного канала. Численное решение, приведенное ниже, получено методами численного интегрирования и переменных модулей при условии плоской деформации изотропной сжимаемой горной породы (рис. 3). При моделировании принято допущение: не учитываются физико-химические процессы взаимодействия бурового раствора с горными породами в приствольной зоне.

\section{Численное решение математической модели}

Упруговязкопластическое перемещение стенки перфорационного канала определяется суммарным упруговязкопластическим перемещением всех элементов в расчетном контуре (1):

$$
U(t)=\sum_{i=1}^{k}\left[\begin{array}{c}
\left(\frac{\mu\left(\Delta \sigma_{r i}+\Delta \sigma_{z i}^{y}\right)-\Delta \sigma_{r i}}{E}\right)+ \\
+\left(\frac{\mu_{t}\left(\Delta \sigma_{t i}+\Delta \sigma_{z i}^{\hat{a i l}}\right)-\Delta \sigma_{r i}}{E_{t}}\right)
\end{array}\right],
$$

где $U(t)$ - упруговязкопластическое перемещение стенки перфорационного канала; $\Delta \sigma_{r i}, \Delta \sigma_{t i}$ - приращение радиального напряжения и тангенциаль- ного напряжения $i$-го элемента массива горных пород соответственно; $\Delta \sigma_{r i}^{y}, \Delta \sigma_{t i}^{\text {sI }}$ - приращение осевого напряжения $i$-го элемента массива горных пород для упругого и вязкопластического деформирования соответственно; $\mu$ - коэффициент Пуассона горной породы; $E$ - модуль упругости горной породы; $\mu_{t}, E_{t}$ - временные функции коэффициента Пуассона и модуля упругости горной породы соответственно [28-30].

Упруговязкопластическое перемещение стенки перфорационного канала определяется отдельно для нижней и верхней стенок, соответствующих точкам А и В на расчетной схеме (рис. 3). При этом формулы для расчета приращения компонент напряжений за боковой стенкой перфорационного канала по линии $A D$ внутри расчетного контура имеют вид (2), (3) [28, 29]:

$$
\begin{gathered}
\Delta \sigma_{\text {б } r i}=p_{\text {б }}-\left(1-\frac{1}{\left(R_{i} / R_{\mathrm{H}}\right)^{2}}\right) \times \\
\times\left[M-N\left(1-\frac{3}{\left(R_{i} / R_{\mathrm{H}}\right)^{2}}\right)\right]-\frac{p_{c}}{\left(R_{i} / R_{\mathrm{H}}\right)^{2}} ; \\
\Delta \sigma_{\text {б } t i}=p_{a}-M\left(1+\frac{1}{\left(R_{i} / R_{\mathrm{H}}\right)^{2}}\right)- \\
-N\left(1+\frac{3}{\left(R_{i} / R_{\mathrm{H}}\right)^{2}}\right)+\frac{p_{c}}{\left(R_{i} / R_{\mathrm{H}}\right)^{2}},
\end{gathered}
$$

где $\Delta \sigma_{\sigma r i}, \Delta \sigma_{\bar{t} t i}-$ приращение радиального и тангенциального напряжения соответственно $i$-го элемента массива горных пород за боковой стенкой; $p_{6}$ - боковое горное давление; $p_{c}$ - гидростатическое давление бурового раствора; $R_{i}, R_{\text {н }}$ расшифрованы в подрисуночной надписи к рис. $3 ; M, N$ - компоненты горного давления в расчетном сечении, определяемые уравнениями (4)-(6): 


$$
p_{a}=p_{\text {г }} \sin ^{2} a+p_{\sigma} \cos ^{2} a,
$$

где параметры $\alpha, p_{r}, p_{a}$ расшифрованы в подрисуночной надписи к рис. 3 [26, 27].

$$
\begin{aligned}
& M=\frac{\left(p_{a}+p_{6}\right)}{2}, \\
& N=\frac{\left(p_{a}-p_{6}\right)}{2} .
\end{aligned}
$$

Формулы для расчета изменения компонент напряжений над верхней стенкой перфорационного канала по линии ВЕ внутри расчетного контура имеют вид (7), (8) [28, 29]:

$$
\begin{gathered}
\Delta \sigma_{\mathrm{Br} i}=p_{a}-\left(1-\frac{1}{\left(R_{i} / R_{\mathrm{H}}\right)^{2}}\right) \times \\
\times\left[M+N\left(1-\frac{3}{\left(R_{i} / R_{\mathrm{H}}\right)^{2}}\right)\right]-\frac{p_{c}}{\left(R_{i} / R_{\mathrm{H}}\right)^{2}} ; \\
\Delta \sigma_{\mathrm{B} t i}=p_{\sigma}-M\left(1+\frac{1}{\left(R_{i} / R_{\mathrm{H}}\right)^{2}}\right)+ \\
+N\left(1+\frac{3}{\left(R_{i} / R_{\mathrm{H}}\right)^{2}}\right)+\frac{p_{c}}{\left(R_{i} / R_{\mathrm{H}}\right)^{2}},
\end{gathered}
$$

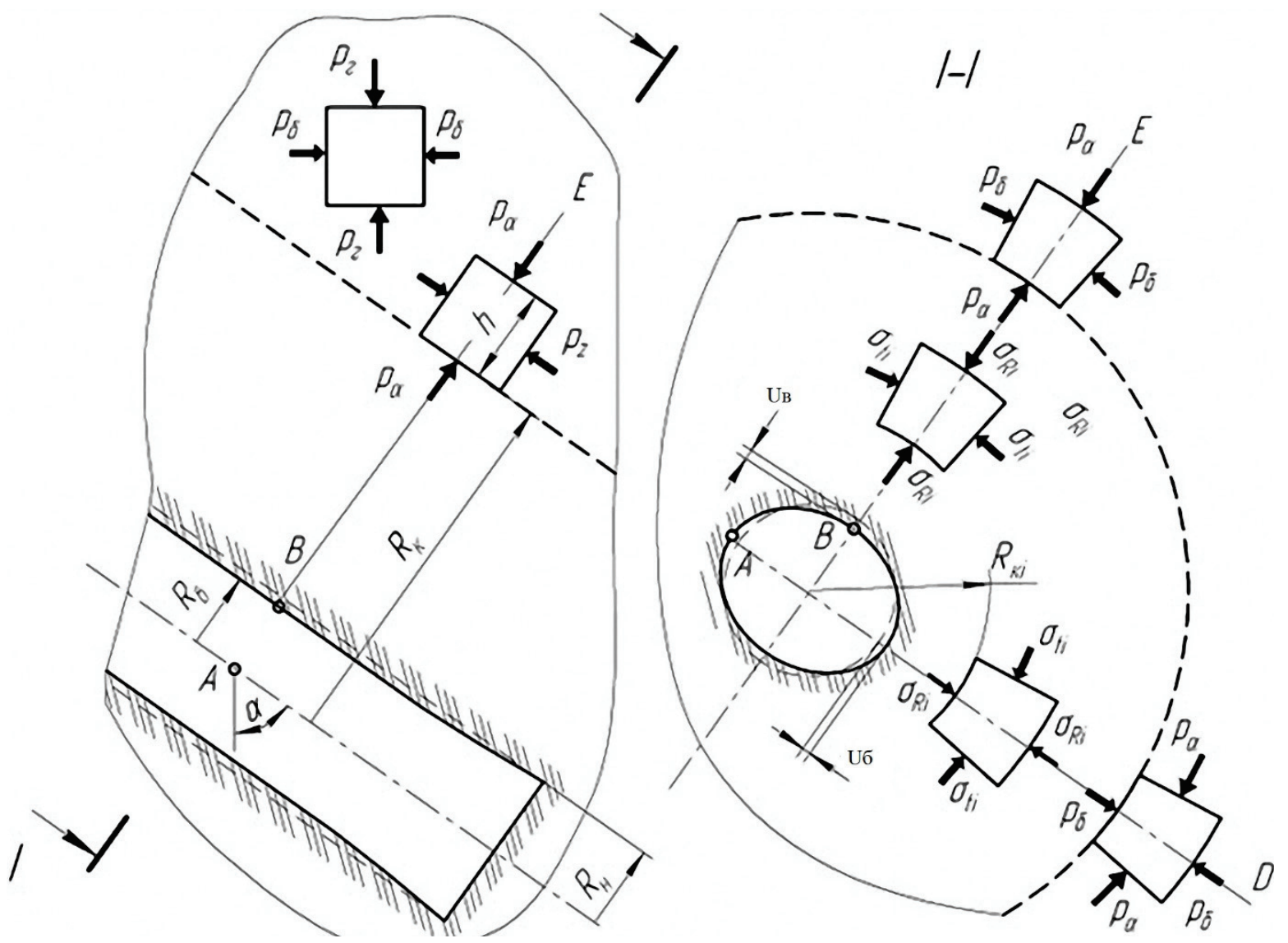

Pис. 3. Расчетная схема перфорационного канала [26, 27]: т.A-боковая стенка перфорационного канала; т. B-верхняя стенка перфорационного канала; т. E, m. D- точки на расчетном контуре в приствольной зоне; $\alpha$ - зенитный угол интервала перфораиионного канала; $p_{2}$ - геостатическое давление; $p_{6}$ - боковое горное давление; $p_{a}$ - давление на i-й элелент лассива горных пород, обусловленное действием геостатического горного давления и бокового горного давления [26]; $h$ - толщина і-го элелента массива горных пород; $\sigma_{r i}, \sigma_{t i}-$ радиальное и тангенииальное напряжение $і$-го элемента массива горных пород соответственно; $R_{i}-$ средний радиус і-го әлемента массива горных пород; $R_{k i}-$ радиус контура i-го элемента лассива горных пород; $R_{н}-$ нолинальный радиус перфорационного канала; $R_{c}$ - фактический радиус перфорационного канала; $R_{k}-$ радиус расчетного контура в приствольной зоне; $U_{\sigma}-$ упруговязкопластическое перелещение боковой стенки перфорационного канала; $U_{\text {в }}-$ упруговязкопластическое перемещение верхней стенки перфорационного канала

Fig. 3. Calculation scheme of the perforation channel $[26,27]: p . A$ - side wall of the perforation channel; $p . B$ - top wall of the perforation channel; p.E, p.D-points on the design contour in the near-well zone; $\alpha$-inclination angle of the interval of the perforation channel; $p_{2}$ - geostatic pressure; $p_{\sigma}$-lateral rock pressure; $p_{a}$ - pressure on the i element of the rock mass, due to the effect of geostatic rock pressure and lateral rock pressure [26]; $h$ - thickness of the i element of the rock massif; $\sigma_{r i}, \sigma_{t i}$-radial stress and tangential stress of the $i$ element of the rock massif, respectively; $R_{i}$ - average radius of the i element of the rock massif, $R_{k i}$ - radius of the contour of the i element of the rock massif; $R_{H}$ - nominal radius of the perforation channel; $R_{c}$ - actual radius of the perforation channel; $R_{k}$ - radius of the design contour in the near-well zone; $U_{\sigma}$ - elastoviscoplastic displacement of the perforation channel side wall; $U_{B}$ - elastoviscoplastic displacement of the perforation channel upper wall 
где $\Delta \sigma_{\text {вri }}, \Delta \sigma_{\text {вti }}-$ приращение радиального и тангенциального напряжения соответственно $i$-го элемента массива горных пород за верхней стенкой.

Формула приращения осевого напряжения для упругого деформирования (9) [29]:

$$
\begin{gathered}
\Delta \sigma_{z i}^{y}=\frac{\left[\frac{(1+\mu)^{2}}{16 E(1-\mu)^{2}(1-2 \mu)}\right]\left(p_{\sigma}-p_{c}\right)}{\left(R_{i} / R_{\mathrm{H}}\right)^{4}}- \\
=\frac{2\left(p_{a}-p_{\sigma}\right)}{\left(R_{i} / R_{\mathrm{H}}\right)^{2}} .
\end{gathered}
$$

Формула приращения осевого напряжения $\Delta \sigma_{z, i}$ для вязкопластического деформирования (10):

$$
\begin{gathered}
\Delta \sigma_{z i}^{\mathrm{BI}}=\frac{\left[\frac{\left(1+\mu_{t}\right)^{2}}{16 E\left(1-\mu_{t}\right)^{2}\left(1-2 \mu_{t}\right)}\right]\left(p_{\sigma}-p_{c}\right)}{\left(R_{i} / R_{\mathrm{H}}\right)^{4}}- \\
-\frac{2\left(p_{a}-p_{\sigma}\right)}{\left(R_{i} / R_{\mathrm{H}}\right)^{2}} .
\end{gathered}
$$

Выполнение расчетов по полученным формулам осуществляется с помощью программного обеспечения, разработанного в среде MS Excel.

\section{Моделирование}

Выполним прогнозирование упруговязкопластического перемещения стенок перфорационного канала после вскрытия его технической системой «Перфобур» на нефтяном месторождении в Поволжье. Терригенный пласт-коллектор представлен переслаиванием алевролита, аргиллита, песчаника и вскрывается при плотности бурового раствора

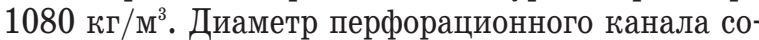
ставляет 60 мм. В таблице приведены исходные данные для моделирования упруговязкопластического перемещения стенок перфорационного канала в интервале продуктивного пласта. Для описания начальной стадии неустановившейся ползучести горных пород применяется ядро Абеля [29].

таблица. Исходные данные для моделирования

Table. $\quad$ Initial data for modeling

\begin{tabular}{|c|c|c|c|c|c|}
\hline $\begin{array}{c}\text { Горная порода } \\
\text { Rосk }\end{array}$ & $\begin{array}{c}E, \mathrm{M \Pi а} \\
\mathrm{MPa}\end{array}$ & $\mu$ & $\begin{array}{c}p_{\mathrm{r}}, \mathrm{M \Pi а} \\
\mathrm{MPa}\end{array}$ & $\begin{array}{c}\delta, \mathrm{c}^{\mathrm{p}-1} \\
\mathrm{~s}^{2 \mathrm{a}-1}\end{array}$ & $f$ \\
\hline $\begin{array}{c}\text { Аргиллит } \\
\text { Argillite }\end{array}$ & 1961,2 & 0,4 & 73,9 & 0,0080 & 0,710 \\
\hline $\begin{array}{c}\text { Алевролит } \\
\text { Siltstone }\end{array}$ & $5883,6 \ldots 7844,8$ & 0,1 & 75,42 & 0,0094 & 0,726 \\
\hline $\begin{array}{c}\text { Песчаник } \\
\text { Sandstone }\end{array}$ & $2941,8 \ldots 8825,4$ & 0,3 & 75,89 & 0,0021 & 0,670 \\
\hline
\end{tabular}

$\delta$, $f$ - параметры ядра Абеля (parameters of Abel kernel) [29, 30] $\mu, E, p_{\text {г }}$ приведены в уравнении (1) (presented in equation (1)).

Отрицательное значение упруговязкопластического перемещения стенок перфорационного канала принимается как сужение перфорационного канала, а положительное значение, соответственно, принимается как расширение перфорационного канала.

В связи с тем, что техническая система «Перфобур» позволяет выполнять радиальные перфо- рационные каналы различного радиуса кривизны, были выполнены расчеты упруговязкопластического перемещения стенок перфорационного канала для максимального широкого диапазона $\alpha=0 \ldots 90^{\circ}$.

На рис. 4 приведена зависимость упруговязкопластического перемещения стенок перфорационного канала от $\alpha$ в пласте аргиллита через один час после вскрытия пласта долотом.

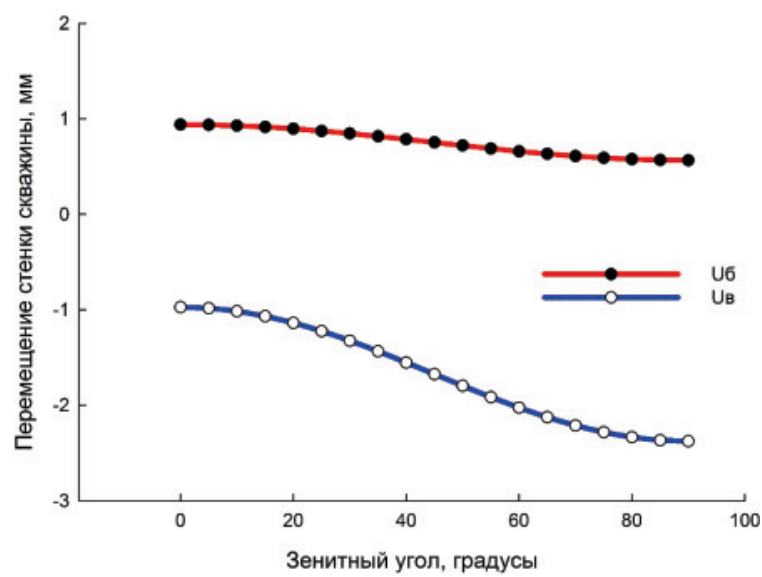

Pис.4. Упруговязкопластическое перемещение стенки перфора иионного канала от $\alpha$ в пласте аргиллита: $U_{6}-$ упруго вязкопластическое перемещение боковой стенки; $U_{\mathrm{B}}$ упруговязкопластическое перемещение верхней стенки

Fig. 4. Elasticviscoplastic displacement of the perforation channel wall from $\alpha$ in the argillite layer: $U_{6}$ - elastoviscoplastic displacement of the perforation channel side wall; $U_{\mathrm{B}}$ - elastoviscoplastic displacement of the perforation channel upper wall

Как видно на рис. 4 , с увеличением $\alpha$ упруговязкопластическое перемещение стенок перфорационного канала возрастает, причем упруговязкопластическое перемещение верхней стенки перфорационного канала в два раза превышает упруговязкопластическое перемещение боковой стенки и противоположно ему по знаку. При $\alpha=0,15^{\circ}$ упруговязкопластическое перемещение стенок перфорационного канала имеет одинаковое по модулю значение, противоположное по знаку $(\approx 1 \mathrm{MM})$.

Зависимость упруговязкопластического перемещения стенок перфорационного канала для пласта алевролита через один час после вскрытия пласта долотом имеет два характерных участка (рис. 5).

Как видно на рис. 5 , при $\alpha=0 . . .15^{\circ}$ упруговязкопластическое перемещение стенок перфорационного канала имеет одинаковое значение по модулю ( $\approx 0,2$ мм). С увеличением $\alpha \succ 45^{\circ}$ упруговязкопластическое перемещение верхней стенки уменьшается до нуля, а затем возрастает с отрицательным значением.

При этом упруговязкопластическое перемещение боковой стенки перфорационного канала возрастает с отрицательным значением. При $\alpha=45^{\circ}$ упруговязкопластические перемещения боковой и верхней стенок перфорационного канала равны. 
С увеличением $\alpha \succ 45^{\circ}$ упруговязкопластические перемещения боковой и верхней стенок перфорационного канала изменяются с отрицательным значением, при этом на верхней стенке упруговязкопластическое перемещение в 1,5 больше, чем на боковой стенке.

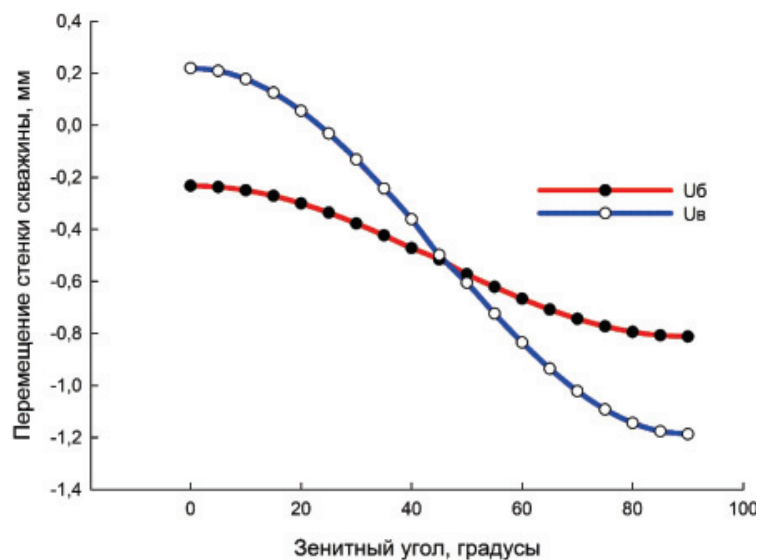

Puc. 5. Упруговязкопластическое перелещение стенки перфораиионного канала от $\alpha$ в пласте алевролита: $U_{6}-$ упруго вязкопластическое перемещение боковой стенки; $U_{\mathrm{B}}$ упруговязкопластическое перемещение верхней стенки

Fig. 5. Elasticviscoplastic displacement of the perforation channel wall from $\alpha$ in the siltstone layer: $U_{6}$ - elastoviscoplastic dis placement of the perforation channel side wall; $U_{\mathrm{B}}$ - elastovis coplastic displacement of the perforation channel upper wall

На рис. 6 показана зависимость упруговязкопластического перемещения стенки перфорационного канала от $\alpha$ для пласта песчаника через один час после вскрытия породы долотом. При $\alpha=0 . . .15$ упруговязкопластическое перемещение верхней и боковой стенок равно по значению и противоположно по знаку. При росте $\alpha \succ 45^{\circ}$ упруговязкопластическое перемещение как на боковой стенке, так и на верхней стенке перфорационного канала увеличивается с отрицательным значением и на верхней стенке упруговязкопластическое перемещение в 6 раз больше аналогичной величины на боковой стенке перфорационного канала.

Максимальные значения упруговязкопластического перемещения стенок перфорационного канала характерны для $\alpha=90^{\circ}$. На рис. 7 представлено изменение во времени упруговязкопластического перемещения стенок перфорационного канала при $\alpha=90^{\circ}$ для аргиллита, алевролита и песчаника. В первые два часа после вскрытия долотом для всех перечисленных горных пород характерен линейный участок роста перемещения стенок перфорационного канала. Далее для алевролита и песчаника кривая упруговязкопластического перемещения стенок перфорационного канала во времени имеет затухающий характер. В то же время для аргиллита упруговязкопластическое перемещение стенок перфорационного канала имеет незатухающий характер. При прочих равных условиях упруговязкопластическое перемещение верхней стенки скважины в пласте аргиллита в 1,5 и
2,2 раза больше, чем в пласте песчаника и алевролита соответственно.

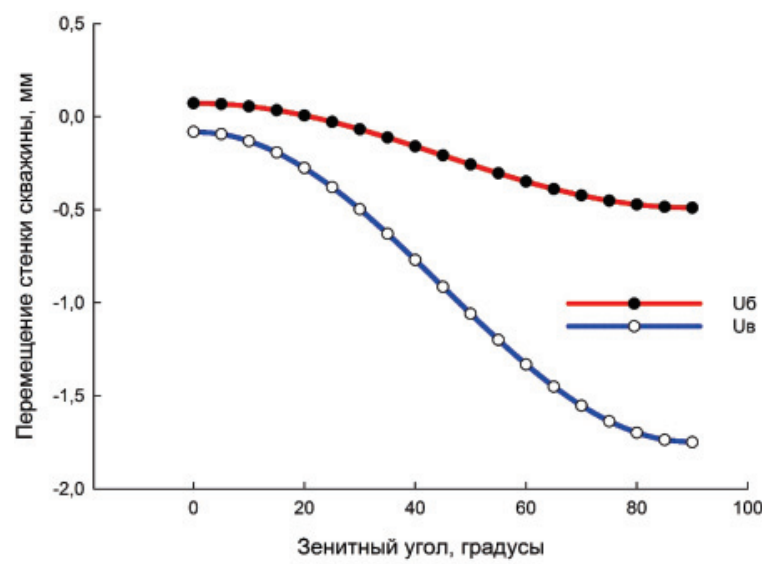

Puc. 6. Упруговязкопластическое перемещение стенки перфора иионного канала от $\alpha$ в пласте песчаника

Fig.6. Elasticviscoplastic displacement of the perforation channel wall from $\alpha$ in the sandstone layer

Выполнен анализ прихватоопасности долот в данном интервале. КНБК ТС оснащается долотами типа PDC. Для долот PDC, исходя из их конструктивных особенностей, принимаем значение опасного радиального упруговязкопластического перемещения стенок перфорационного канала, равное 2 мм, при котором возможно заклинивание при спускоподъемных операциях. Отсюда, по рис. 4-7, видно, что заклинивание породоразрушающего инструмента произойдет в интервале аргиллита при $\alpha \succ 55^{\circ}$ уже через час после вскрытия горной породы. Таким образом, время безопасного ведения работ составляет один час.

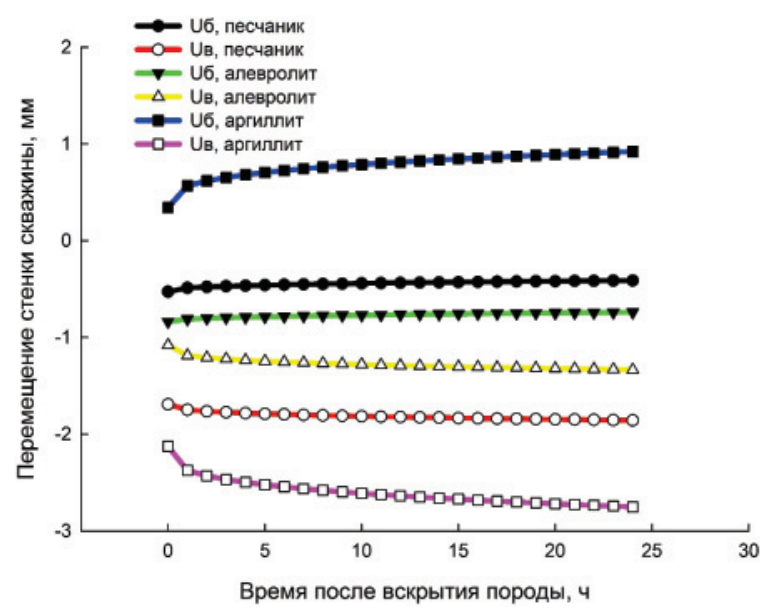

Pис. 7. Упруговязкопластическое перемещение стенки перфора иионного канала $\left(\alpha=90^{\circ}\right)$ во врелени

Fig. 7. Elasticviscoplastic displacement of the perforation channel wall $\left(\alpha=90^{\circ}\right)$ in time

Поэтому, кроме борьбы с возможными дифференциальными прихватами, рассмотренными в начале статьи, ведутся исследования по разработке способов предупреждения заклинивания долота технической системы «Перфобур» в опасном ин- 


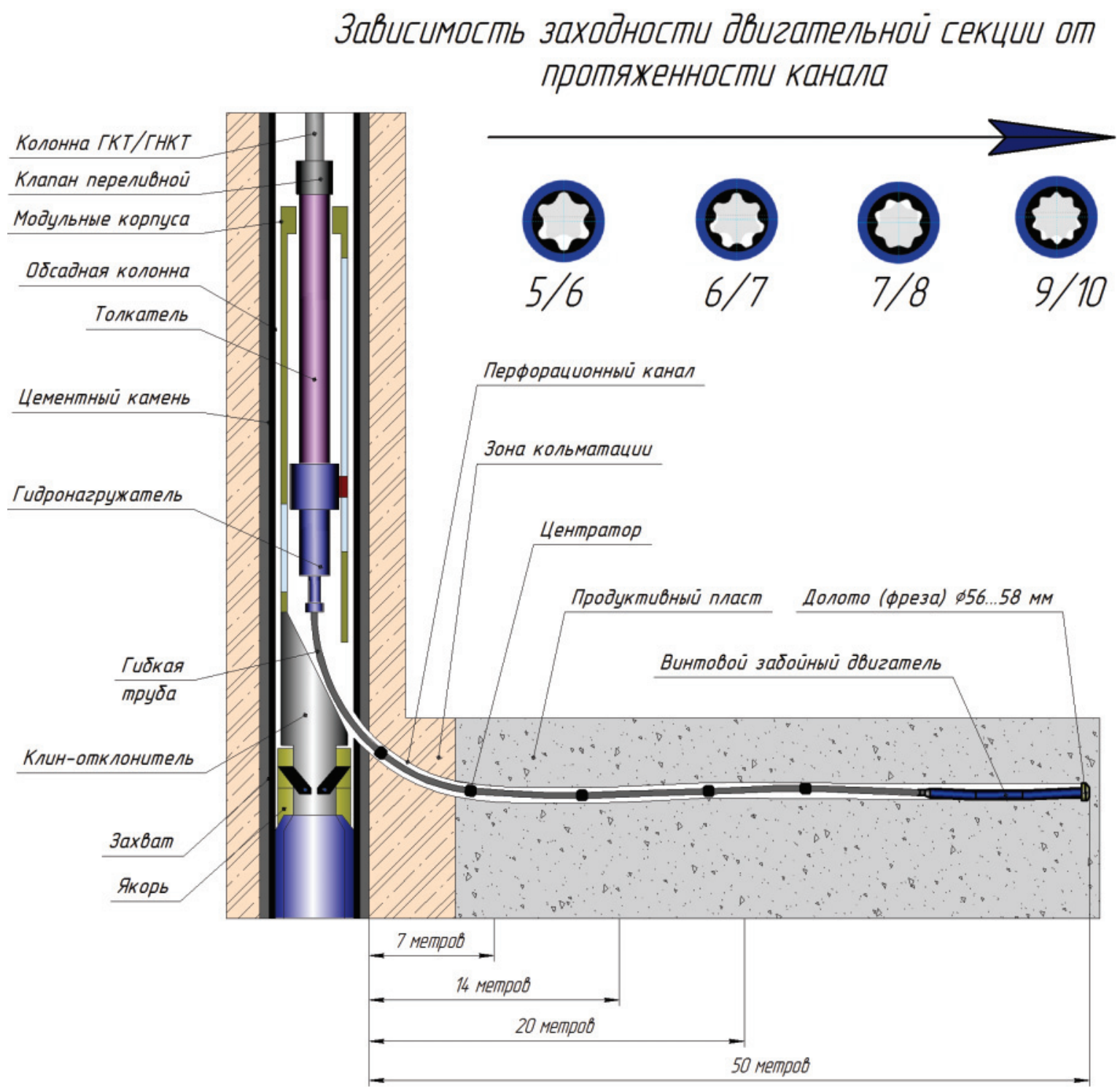

Pис. 8. Рекомендуемые винтовые забойные двигатели (ВЗД) с различными кинематическими соотношенияли рабочих органов для колпоновок ТС «Перфобур» при бурении перфорационных каналов различной протяженности

Fig. 8. Recommended screw downhole motors with different kinematic ratio of operating elements for the technical system assembly «Perfobur» for drilling perforation channels with different length

тервале. Изменение гидростатического давления бурового раствора ограничено условиями устойчивости горных пород и предупреждения поглощения бурового раствора. Компоновки технической системы «Перфобур» в настоящее время модернизированы путем добавления специальных устройств модульного типа для предупреждения рассматриваемых осложнений [3-6, 31, 32]. Одним из рекомендуемых способов предупреждения прихватов является увеличение механической скорости бурения [33, 34]. На рис. 8 представлены КНБК с рекомендуемыми вариантами кинематики рабочих органов винтового забойного двигателя (ВЗД) для бурения перфорационных каналов различной протяженности при оптимальной механической скорости.
Сокращение сроков бурения радиальных каналов также позволяет уменьшить риски осложнений. В настоящее время для компании «Перфобур» по разработанному техническому заданию изготовлен новый двухсекционный винтовой забойный двигатель габарита 49 мм с кинематическим отношением рабочих органов (РО) 9:10, что позволило кратно увеличить механическую скорость бурения по сравнению с ранее применяемыми ВЗД габарита 43 мм, с кинематикой PO - 5:6, и бурить перфорационный канал длиной 7-14 м за 49 минут. Работы по совершенствованию ВЗД ведутся на постоянной основе.

\section{Выводы}

1. По результатам численного моделирования напряженно-деформированного состояния гор- 
ных пород после бурения глубокого перфорационного канала в терригенном коллекторе месторождения в Поволжье определены значения упруговязкопластического перемещения его стенок при вариации зенитного угла в диапазоне $0^{\circ} \leq \alpha \leq 90^{\circ}$ и во времени после вскрытия породы при $\alpha=90^{\circ}$.

2. Анализ полученных расчетных значений упруговязкопластического перемещения стенок глубокого перфорационного канала показал, что наиболее опасным с точки зрения заклинивания инструмента при выполнении спускоподъемных операций является интервал аргиллита, где время безопасного ведения работ составляет один час.

\section{СПИСОК ЛИТЕРАТУРЫ}

1. Dickinson W., Anderson R.R., Dickinson R.W. The UltrashortRadius Radial System // SPE Drilling Engineering. - 1989. V. 4 (3). - P. 247-254.

2. Электронные ресурсы фирмы Radial Drilling Services. URL: https://www.radialdrilling.com/ (дата обращения: 07.09.2018).

3. Мальцев А.А., Лягов И.А., Лягов А.В. Разработка инновационной системы радиального бурения для повышения нефтеотдачи пласта // Нефть. Газ. Новации. - 2016. - № 11. C. 64-67.

4. Analytical research and experimental tests on the technology for drilling small diameter channels with small radius of curvature / I.A. Lyagov, N.I. Vasilev, M. Reich, M. Mezzetti // Oil Gas European Magazine. - 1 September 2014. - V. 40. - Iss. 3. P. 124-129.

5. Lyagov I.A. Bottomhole formation zone completion through ultra deep multibranch channels experimental research of a new technology // Mine planning and equipment selection. Proc. of the 22 $2^{\text {nd }}$ MPES Conference. - Dresden, Germany: Springer, 2013. - P. 1221-1229.

6. Лягов И.А., Лягова М.А. Технология вскрытия продуктивных пластов скважины бурением каналов малого диаметра и радиуса кривизны / Сборник материалов конференции и выставки SPE по разработке месторожждений в осложненных условиях и Арктике. - M.: Society of Petroleum Engineers, 2013. C. 835-873.

7. Caenn R., Darley H.C.H, Gray G.R. Composition and Properties of Drilling and Completion Fluids. $7^{\text {th }}$ ed. - Cambridge: Gulf Professional Publishing, 2016. $-748 \mathrm{p}$.

8. DeGeare J. The Guide to Oilwell Fishing Operations. Tools, Techniques, and Rules of Thumb. $2^{\text {nd }}$ ed. - Cambridge: Gulf Professional Publishing, 2014. - $234 \mathrm{p}$.

9. Самотой А.К. Предупреждение и ликвидация прихватов труб при бурении скважин. - М.: Недра, 1979. - 182 с.

10. Amadei B., Stephansson 0. Rock stress and its measurement. Dordrecht: Springer Science+Business Media, 1997. - 276 p.

11. Amadei B. Rock Anisotropy and the Theory of Stress Measurements. - Berlin; Heidelberg: Springer-Verlag, 1983. - 482 p.

12. Brady B.H.G., Brown E.T. Rock Mechanics for underground mining. $3^{\text {rd }}$ ed. - Dordrecht: Kluwer Academic Publishers, 2004. $645 \mathrm{p}$.

13. Civa F. Reservoir Formation Damage Fundamentals, Modeling, Assessment, and Mitigation. ${ }^{\text {rd }}$ ed. - Cambridge: Gulf Professional Publishing, Elsevier, 2016. - 1044 p.

14. Jaeger J.C., Cook N.G.W., Zimmerman R.W. Fundamentals of Rock Mechanics. $4^{\text {th }}$ ed. - Malden: Blackwell Publishing, 2007. $489 \mathrm{p}$.
3. Для предупреждения заклинивания технической системы «Перфобур» в неустойчивых интервалах предлагается:

a) применять регламентированные по времени проработки пробуренных интервалов ствола перфорационного канала;

б) использовать в КНБК специальные устройства (демпфер-осциллятор, калибратор, расширитель, кольмататор), устанавливаемые в компоновку в расчетных местах;

в) увеличить механическую скорость бурения перфорационного канала за счет использования специальных винтовых забойных двигателей с рекомендуемыми кинематическими отношениями рабочих органов в зависимости от длины канала.

15. Goodman R.E. Introduction of Rock Mechanics. $2^{\text {nd }}$ ed. - Hoboken: John Wiley \& Sons, 1989. - $562 \mathrm{p}$.

16. Hudson J.A., Harrison J.P. Engineering rock mechanics. An introduction to the principles. - Oxford: Elsevier, 1997. - 497 p.

17. Hencher S. Practical Rock Mechanics. - Boca Raton: CRC Press, Taylor \& Francis Group, 2015. - $371 \mathrm{p}$.

18. Jaeger C. Rock Mechanics and Engineering. - Cambridge: Cambridge University Press, 1972. - $256 \mathrm{p}$.

19. Parry R.H.G. Mohr Circles, Stress Paths and Geotechnics. $2^{\text {nd }}$ ed. New York: Taylor \& Francis Group, 2004. - 279 p.

20. Pusch R. Rock Mechanics on a Geological Base. - Oxford: Elsevier, 1995. $-519 \mathrm{p}$.

21. Small J.C. Geomechanics in soil, rock, and environmental engineering. - Boca Raton: CRC Press, Taylor \& Francis Group, 2017. $560 \mathrm{p}$.

22. Aadnoy B.S., Larsen K., Berg C. Analysis of stuck pipe in deviated boreholes // Journal of Petroleum Science and Engineering. March 2003. - V. 37. - Iss. 3-4. - P. 195-212.

23. Wellbore stability analysis based on a new strength criterion / X. Shi, W. Cai, Y. Meng, G. Li, J. Li // Journal of Natural Gas Science and Engineering. - November 2015. - V. 27. - P. 2. P. $1005-1015$.

24. Kanfar M.F., Chen Z., Rahman S.S. Effect of material anisotropy on time-dependent wellbore stability // International Journal of Rock Mechanics and Mining Sciences. - September 2015. V. 78 . - P. 36-45.

25. Wellbore stability analysis and breakout pressure prediction in vertical and deviated boreholes using failure criteria - a case study / M. Mansourizadeh, M. Jamshidian, P. Bazargan, 0. Mohammadzadeh // Journal of Petroleum Science and Engineering. - September 2016. - V. 145. - P. 482-492.

26. Zeynali M.E. Mechanical and physico-chemical aspects of wellbore stability during drilling operations // Journal of Petroleum Science and Engineering. - February-March 2012. - V. 82-83. P. 120-124.

27. Kanfar M.F., Chen Z., Rahman S.S. Risk-controlled wellbore stability analysis in anisotropic formations // Journal of Petroleum Science and Engineering. - October 2015. - V. 134. - P. 214-222.

28. Попов А.Н., Могучев А.И., Попов М.А. Деформирование стенок наклонной скважины и его влияние на работу и изнашивание буровых долот / Строительство нефтяных и газовых скважин на суше и на море. - 2008. - № 3. - С. 6-13.

29. Расчет упруговязкопластического перемещения стенки открытого ствола скважины с учетом сжимаемости горной породы / А.Г. Губайдуллин, А.И. Могучев, В.У. Ямалиев, А.В. Лягов // Нефтяное хозяйство. - 2018. - № 2. - С. 53-56.

30. Амусин Б.З., Линьков А.М. Об использовании метода переменных модулей для решения одного класса задач линейной на- 
следственной ползучести // Известия АН СССР. Механика твердого тела. - 1974. - № 6. - С. 162-166.

31. Ержанов Ж.С. Теория ползучести горных пород и ее приложения. - Алма-Ата: Наука, 1964. - 173 с.

32. Кацауров И.Н. Механика горных пород. - М.: Недра, 1981. $166 \mathrm{c.}$
33. Reich Matthias. Auf krummen Touren durch den Untergrund ein Exkurs in die Welt der Richtbohrtechnik // Erdöl, Erdgas, Kohle, - Januar 2011. - № 130. - P. 35-41.

34. Bradley W.B. Factors affecting the control of borehole angle in straight and directional wells // Journal of petroleum technology. - 1975. - V. 27. - № 6. - P. 679-688.

Поступила 26.11.2018 2.

\section{Информация об авторах}

Лягов И.А., кандидат технических наук, генеральный директор 000 «Перфобур» .

Губайдуллин A.Г., кандидат технических наук, ассистент кафедры машин и оборудования нефтегазовых промыслов Горно-нефтяного факультета Уфимского государственного нефтяного технического университета.

Ллгов А.B., доктор технических наук, профессор кафедры машин и оборудования нефтегазовых промыслов Горно-нефтяного факультета Уфимского государственного нефтяного технического университета; директор по научно-исследовательским и опытно-конструкторским работам 000 «Перфобур».

Могучев А.И., кандидат технических наук, доцент кафедры машин и оборудования нефтегазовых промыслов Горно-нефтяного факультета Уфимского государственного нефтяного технического университета.

Попов А.H., доктор технических наук, профессор кафедры бурения нефтяных и газовых скважин Горно-нефтяного факультета Уфимского государственного нефтяного технического университета. 


\section{FORECASTING THE RISKS OF JAMMING TO EXCLUDE THE POSSIBILITY OF STITCHING THE TECHNICAL SYSTEM «»PERFOBUR» WHILE DRILLING BRANCHED CHANNELS IN TERRIGENOUS RESERVOIRS}

llya A. Lyagov',

ilyagov@perfobur.com

Azat G. Gubaidullin², azat771989@mail.ru

Alexander V. Lyagov ${ }^{2,1}$, lyagovs@mail.ru

Alexander I. Moguchev², moguchev@rusoil.net

Anatoly N. Popov ${ }^{2}$,

ngpo_ugntu@mail.ru

1 Perfobur,

bld. 2, 7, Derbenevskaya Embankment, Moscow, 115114, Russia.

2 Ufa state petroleum technical university,

1, Kosmonavtov street, Ufa, 450062, Russia.

The relevance of the research. One of the promising methods of secondary formation exposing is drilling of deep perforations by the "Perfobur» radial drilling system. This article discusses the issues of forecasting and preventing freeze-in of the technical system «Perfobur» due to bit stall in the intervals of incompetent rock. The bit stall in these intervals occur due to the elastoviscoplastic deformation of the perforation channel cross section.

The aim of the research is to evaluate the risks of sticking of the technical system «Perfobur» due to bit stall during the tripping operations and to develop the measures to prevent them.

Objects: deep perforation channel in the terrigenous reservoir of oil field in the Volga region, which is an alternation of mudstone, siltstone and sandstone.

Methods: geomechanical modeling, numerical integration, variable module method.

Results. The authors have determined the movement of the deep perforation channel walls in time for the terrigenous reservoir, which is an alternation of argillite, siltstone and sandstone and obtained the dependences of the elastoviscoplastic movement of the perforation channel walls with changes in inclination angle. The elasticviscoplastic displacement of the perforation channel upper wall, as a rule, considerably (by 1,5 or more times) exceeds the elastoviscoplastic displacement of the perforation channel side wall. Consequently, the elasticviscoplastic displacement of the perforation channel upper wall is the most dangerous and determining from the point of view of bit stall during the tripping operations. Based on the established criterion of the PDC bit stall (dangerous displacement of the wall is equal to $2 \mathrm{~mm}$ ), it is determined that the most dangerous from the point of view of bit stall is the argillite deposit and the time of safe work is one hour. To reduce the risk of the bit stall of the technical system "Perfobur», it was proposed to use a new screw downhole motor PDM-49, as well as the stabilizers, expanders, calibrators, and colmatators in perforation to prevent the elimination of possible complications in the perforation channel.

\section{Key words:}

Technical system «Perfobur», bottom hole assembly, bit seizure, elastoviscoplastic deformation of rock, bottomhole formation zone, drilling of branched radial perforation channels.

\section{REFERENCES}

1. Dickinson W., Anderson R.R., Dickinson R.W. The UltrashortRadius Radial System. SPE Drilling Engineering, 1989, vol. 4 (3), pp. 247-254.

2. Electronic resources of the company Radial Drilling Services. Available at https://www.radialdrilling.com/ (accessed $7 \mathrm{Se}$ ptember 2018)

3. Maltsev A.A., Lyagov I.A., Lyagov A.V. Development of innovative radial drilling system to enhance oil recovery. Oil. Gas. Innovations, 2016, no. 11, pp. 64-67. In Rus.

4. Lyagov I.A., Vasilev N.I., Reich M., Mezzetti M. Analytical research and experimental tests on the technology for drilling small diameter channels with small radius of curvature. Oil Gas
European Magazine, 1 September 2014, vol. 40, Iss. 3, pp. 124-129.

5. Lyagov I.A. Bottomhole formation zone, completion of ultra deep multibranch channels, experimental research of a new technology. Mine planning and equipment selection. Dresden, Germany, Springer, 2013. pp. 1221-1229.

6. Lyagov I.A., Lyagova M.A. Tekhnologiya vskrytiya produktivnykh plastov skvazhiny bureniem kanalov malogo diametra i radiusa krivizny [Technology of productive wells formation exposing by channels drilling with small diameter and the radius of curvature]. Sbornik materialov konferentsii i vystavki SPE po razrabotke mestorozhdeniy v oslozhnennykh usloviyakh $i$ Arktike [SPE Conference Paper of «SPE Arctic \& Extreme Environ- 
ments»]. Moscow, Society of Petroleum Engineers Publ., 2013. pp. 835-873.

7. Caenn R., Darley H.C.H, Gray G.R. Composition and Properties of Drilling and Completion Fluids. $7^{\text {th }}$ ed. Cambridge, Gulf Professional Publishing, 2016. - $748 \mathrm{p}$.

8. DeGeare J. The Guide to Oilwell Fishing Operations. Tools, Techniques, and Rules of Thumb. $2^{\text {nd }}$ ed. Cambridge, Gulf Professional Publishing, 2014. 234 p.

9. Samotoy A.K. Preduprezhdenie i likvidatsiya prikhvatov trub pri burenii skvazhin [Prevention and elimination of the drill pipe freeze-in for the well-drilling]. Moscow, Nedra Publ., 1979. 182 p.

10. Amadei B., Stephansson 0. Rock stress and its measurement. Dordrecht, Springer Science+Business Media, 1997. 276 p.

11. Amadei B. Rock Anisotropy and the Theory of Stress Measurements. Berlin; Heidelberg, Springer-Verlag, 1983.482 p.

12. Brady B.H.G., Brown E.T. Rock Mechanics for underground mining. $3^{\text {rd }}$ ed. Dordrecht, Kluwer Academic Publishers, 2004. $645 \mathrm{p}$.

13. Civa F. Reservoir Formation Damage Fundamentals, Modeling, Assessment, and Mitigation. $3^{\text {rd }}$ ed. Cambridge, Gulf Professional Publishing, Elsevier, 2016. 1044 p.

14. Jaeger J.C., Cook N.G.W., Zimmerman R.W. Fundamentals of Rock Mechanics. $4^{\text {th }}$ ed. Malden, Blackwell Publishing, 2007. $489 \mathrm{p}$.

15. Goodman R.E. Introduction of Rock Mechanics. $2^{\text {nd }}$ ed. Hoboken, John Wiley \& Sons, 1989. $562 \mathrm{p}$.

16. Hudson J.A., Harrison J.P. Engineering rock mechanics. An introduction to the principles. Oxford, Elsevier, 1997. $497 \mathrm{p}$.

17. Hencher S. Practical Rock Mechanics. Boca Raton, CRC Press, Taylor \& Francis Group, 2015. $371 \mathrm{p}$.

18. Jaeger C. Rock Mechanics and Engineering. Cambridge, Cambridge University Press, 1972. $256 \mathrm{p}$.

19. Parry R.H.G. Mohr Circles, Stress Paths and Geotechnics. $2^{\text {nd }}$ ed. New York, Taylor \& Francis Group, 2004. 279 p.

20. Pusch R. Rock Mechanics on a Geological Base. Oxford, Elsevier, 1995. $519 \mathrm{p}$.

21. Small J.C. Geomechanics in soil, rock, and environmental engineering. Boca Raton, CRC Press, Taylor \& Francis Group, 2017. $560 \mathrm{p}$.

22. Aadnoy B.S., Larsen K., Berg C. Analysis of stuck pipe in deviated boreholes. Journal of Petroleum Science and Engineering, March 2003, vol. 37, Iss. 3-4, pp. 195-212.

23. Shi X., Cai W., Meng Y., Li G., Li J. Wellbore stability analysis based on a new strength criterion. Journal of Natural Gas Science and Engineering, November 2015, vol. 27, P. 2, pp. 1005-1015.

\section{Information about the authors}

Ilya A. Lyagov, Cand. Sc., general director, Perfobur.

Azat G. Gubaidullin, Cand. Sc., assistant, Ufa state petroleum technical university.

Alexander V. Lyagov, Dr. Sc., professor, Ufa state petroleum technical university; Research \& Development Director, Perfobur.

Alexander I. Moguchev, Cand. Sc., associate professor, Ufa state petroleum technical university.

Anatoly N. Popov, Dr. Sc., professor, Ufa state petroleum technical university.
24. Kanfar M.F., Chen Z., Rahman S.S. Effect of material anisotropy on time-dependent wellbore stability. International Journal of Rock Mechanics and Mining Sciences, September 2015, vol. 78, pp. $36-45$.

25. Mansourizadeh M., Jamshidian M., Bazargan P., Mohammadzadeh 0 . Wellbore stability analysis and breakout pressure prediction in vertical and deviated boreholes using failure criteria - a case study. Journal of Petroleum Science and Engineering, September 2016, vol. 145, pp. 482-492. re stability during drilling operations. Journal of Petroleum Science and Engineering, February-March 2012, vol. 82-83, pp. 120-124.

27. Kanfar M.F., Chen Z., Rahman S.S. Risk-controlled wellbore staScience and Engineering, October 2015, vol. 134, pp. 214-222.

28. Popov A.N., Moguchev A.I., Popov M.A. Deformation of an inclined well walls and its influence on the work and wear of drill bits. Construction of oil and gas wells on onshore and offshore, 2008, no. 3, pp. 6-13. In Rus.

29. Gubaidullin A.G., Moguchev A.I., Yamaliev V.U., Lyagov A.V. Calculation of the elastoviscoplastic displacement of the open wellbore wall with allowance for the compressibility of the rock. Oil Industry, 2018, no. 2, pp. 53-56. In Rus.

30. Amusin B.Z., Linkov A.M. Ob ispolzovanii metoda peremennykh moduley dlya resheniya odnogo klassa zadach lineynoy nasledstvennoy polzuchesti [On use of the method of variable modules for solving a class of linear hereditary creep problems]. Izvestiya AN SSSR. Mekhanika tverdogo tela, 1974, no. 6, pp. 162-166.

31. Erzhanov Zh.S. Teoriya polzuchesti gornysh porod i ee prilozheni$y a$ [The rocks creep theory and its applications]. Alma-Ata, Nauka Publ., 1964. $173 \mathrm{p}$.

32. Katsaurov I.N. Mekhanika gornykh porod [Rocks Mechanics]. Moscow, Nedra Publ., 1981. 166 p.

33. Reich Matthias. On crooked tours through the underground - an excursion into the world of directional drilling technology. Oil Gas European Magazine, January 2011, vol. 130, pp. 35-41. In German.

34. Bradley W.B. Factors affecting the control of borehole angle in straight and directional wells. Journal of petroleum technology, 1975, vol. 27, no. 6, pp. 679-688.

Received: 26 November 2018.
26. Zeynali M.E. Mechanical and physico-chemical aspects of wellbobility analysis in anisotropic formations. Journal of Petroleum 